\title{
The Impact of Nanoscience on Heterogeneous Catalysis
}

\author{
Alexis T. Bell \\ Division of Chemical Sciences, Lawrence Berkeley National Laboratory and \\ Department of Chemical Engineering, University of California, Berkeley, CA 94720-1462 \\ bell@cchem.berkeley.edu
}

\begin{abstract}
Most catalysts consist of nanometer-sized particles dispersed on a high-surface area support. Advances in characterization methods have led to a molecular level understanding of the relationships between nanoparticle properties and catalytic performance. Together with novel approaches to nanoparticle synthesis, this knowledge is contributing to the design and development of new catalysts.
\end{abstract}

Inexpensive transportation fuels, high-temperature lubricants, chlorine-free refrigerants, high-strength polymers, stain-resistant fiber, cancer-treatment drugs, and many thousands of other products required by modern societies would not be possible without the existence of catalysts. These critical materials mediate the pathway by which chemical reactions occur, enabling highly selective formation of desired products at rates that are commercially viable. Catalysts are essential, as well, for the reduction of air and water pollution, and contribute thereby to reducing the emissions of products that are harmful to human health and the environment. A recent article discussing the economic contributions of catalysis noted that "... one third of material gross national product in the US involves a catalytic a catalytic process somewhere in the production chain" (1).

The majority of the industrial catalysts are high-surface area solids on to which an active component is dispersed in the form of very small particles. These moieties have dimensions of 1-20 $\mathrm{nm}$ and are often referred to as nanoparticles. To illustrate the importance of dispersed nanoparticles, one need only look inside the automotive converter found under the floor of every new car manufactured in the US since the early 1970's. Figure 1 shows that the converter consists of a honeycomb the walls of which are coated with a thin coating of porous alumina. The alumina washcoat is impregnated with nanoparticles of $\mathrm{Pt}, \mathrm{Rh}, \mathrm{Ce}$, zirconia, and lanthana, and occasionally baria. The $\mathrm{Pt}$ serves to oxidize hydrocarbons and carbon monoxide, and the $\mathrm{Rh}$, to reduce $\mathrm{NO}_{\mathrm{x}}$. The ceria, particularly in combination with zirconia, works as an oxygen storage component, 
enabling the oxidation of hydrocarbons and carbon monoxide to occur during moments when the engine exhaust is fuel rich. The lanthana serves to stabilize the alumina against a loss of surface area and the baria acts as a trap for sulfur trioxide - a catalyst deactivator, or "poison."

The importance of small particles to the performance of catalysts has stimulated extensive efforts to develop tools for their characterization $(2,3)$. Originating from the fields of physics, chemistry, materials science, and chemical engineering, this are of study is now often referred to as nanoscience. The aim of the present review is to show how the local size and composition of catalyst particles affects their performance (i.e., activity and selectivity) and to show how advances in nanoscience have contributed to a detailed understanding of the effects of particle composition, size, and structure on catalyst performance. It will also be shown that advances in synthetic methods are leading to increasingly precise control of the variables affecting catalyst activity and selectivity.

Particles in the size range of roughly 1-50 nm exhibit physical and chemical properties that are intermediate between those of the smallest element from which they can be comprised (e.g., a metal atom, the stoichiometric unit of a metal oxide). Catalyst performance can be sensitive to particle size because the surface structure and electronic properties can change greatly in this size range. For example, the heat of adsorption for $\mathrm{CO}$ and the activation energy for $\mathrm{CO}$ dissociation both change with increasing size of $\mathrm{Ni}$ particles, patterns that affect the performance of Ni nanoparticles for Fischer-Tropsch synthesis of hydrocarbons from synthesis gas (a mixture of $\mathrm{CO}$ and $\mathrm{H}_{2}$ ).

Titania supported gold particles have been found to be active at ambient conditions for the oxidation of $\mathrm{CO}$ to $\mathrm{CO}_{2}$, and can be used to reduce the levels of $\mathrm{CO}$ pollution in buildings by incorporating such particles into the paint used to cover the interior walls (4). Figure 2 shows that the activity of the gold particles is very sensitive to their size, and that only particles in the range of 2-3 nm are active (5). This effect has been ascribed to oxidation of the Au atoms that are in contact with the support, and nicely illustrates the importance of tuning the electronic properties of the metal particles to achieve high catalytic activity. 
Nanoparticles of vanadia supported on various oxides $\left(\mathrm{ZrO}_{2}, \mathrm{Al}_{2} \mathrm{O}_{3}, \mathrm{MgO}\right)$ are active catalysts for the oxidative dehydrogenation (ODH) of alkanes to olefins (6). Recent research has shown that the ODH activity per atom of exposed vanadium increases with increasing size of the vanadia particles (7). Studies of the electronic properties of dispersed vanadia show that this trend is associated with the reduction in the ease of $\mathrm{O}$ to $\mathrm{V}$ electron transfer, and hence to the ease of reduction of the vanadia by adsorbed alkane molecules, a critical first step in the ODH chemistry. Consistent with this explanation, the ODH turnover frequency increases with decreasing UV-visible absorption edge energy.

Local composition, as well as particle size, can affect the performance of catalysts. For example, the catalyst used to remove sulfur from petroleum and petroleum products consists of thin layers of $\mathrm{MoS}_{2}$ dispersed on alumina. Decoration of the edge of the $\mathrm{MoS}_{2}$ layers with a small amount of Co increases the desulfurization activity of the catalyst by nearly an order of magnitude (8). Knowledge of the size and shape of the $\mathrm{MoS}_{2}$ particles has come from high-resolution transmission electron microscopy (TEM) whereas information about the location of the Co atoms has come from EXAFS (extended x-ray absorption fine structure) experiments.

TEM together with solid-state NMR has demonstrated that Ru particles deposited on titania are partially covered by patches of amorphous titania. At the adlineation between the metal and the oxide, highly active sites are formed for the Fischer-Tropsch synthesis of hydrocarbons from $\mathrm{H}_{2}$ and $\mathrm{CO}$ (9). By regulating the coverage of the metal by the amorphous oxide, not only can the activity be controlled but also the selectivity of the products to form higher molecular weight olefins. These effects are shown in Fig. 3.

As shown by the preceding illustrations, considerable insight into the factors affecting catalyst activity and selectivity becomes available can be deduced from knowledge of the local composition and structure of catalyst nanoparticles. Since the structure and composition of such particles are known to change under reaction conditions, it is particularly important to characterize catalysts under conditions that are close to those of actual use. As note below, remarkable advances have been made in this direction. 
One of the most informative characterization techniques is TEM, since the electrons passing through the sample provide information not only about particle size and shape, but also about the lattice structure and the chemical composition of individual particles. Advances in controlled environment stages and electron optics now make it possible to examine catalysts at temperatures up to $1000^{\circ} \mathrm{C}$ and pressures up to $101 \mathrm{kPa}$ (10). Such capabilities have been used to show how the shape of metal particles is dictated by interactions of the particles with the support, as well as showing how portions of the support migrate to the surface of the metal particles. From selected-area electron diffraction measurements, it is possible to determine the crystal lattice parameters of individual nanoparticles. The recent advent of electron tomography is adding the capability to define and examine particle shapes in situ (11). Finally, advances in computer processing of the information gathered from an electron microscope are opening the door to the detailed examination of particles as small as several atoms (12).

Scanning tunneling microscopy (STM) and atomic force microscopy (AFM), two of the newer techniques for the characterization of nanoparticles, has been used to examine the nanostructure of model catalyst systems. Using STM, for example, it has been possible to explain why the addition of small amounts of $\mathrm{Au}$ to Ni catalysts used for the steam reforming of methane reduces the tendency of such catalysts to deactivate due to carbon deposition (13). The STM micrograph in Fig. 4 shows Au atoms located on the surface of a $\mathrm{Ni}$ surface. The presence of the $\mathrm{Au}$ atoms near a $\mathrm{Ni}$ atom reduces the tendency of carbon to bond to the $\mathrm{Ni}$, and hence, to initiate the formation of carbonaceous deposits that deactivate the catalyst. Using AFM, it has been possible to image growing polyethylene chains formed on individuals $\mathrm{Cr}$ atoms supported on silica (14). Another area that has seen growth is the use of synchrotron radiation to characterize the local composition and structure of small catalyst particles. For example, the development of capabilities to acquire EXAFS data very rapidly (QEXAFS) has made it possible to observe the changes that occur in the structure of heteropolymetallate acids during their break-in (15). EXAFS has also been used to obtain evidence for the surface enhancement of one metal on the surface of another in bimetallic catalysts. Synchrotron radiation has been used as well to follow changes in the composition of oxide catalysts as they are placed under reaction conditions. With a specially designed XPS cell, 
measurements can now be made at pressures up to 20 Torr and temperatures of up to 673 $\mathrm{K}$ (16). An investigation of methane oxidation over $\mathrm{Cu}_{2} \mathrm{O}$ using such capabilities has demonstrated that the oxide undergoes a partial reduction in situ and that the composition of the products formed is related to the oxidation state of the catalyst.

Advances in the synthesis of materials are leading to exciting opportunities for the creating catalyst particles that are all of the same size and shape. Likewise it is becoming increasingly possible to produce well defined porous matrices, which can serve as catalyst supports, from a wide variety of inorganic materials. These capabilities are contributing to the production of single-site catalysts, in which all of the active sites closely resemble each other. Several illustrations will be given to show what can be done.

Single atoms dispersed onto a high surface area oxide are catalytically active. Zirconium and other transition metal hydrides are quite active for the cleavage of $\mathrm{C}-\mathrm{H}$ and $\mathrm{C}-\mathrm{C}$ bonds $(17,18)$. The later process has made it possible to carry out the metathesis of alkanes. As shown in Fig. 5, molecular precursors can also be used to anchor individual Fe atoms onto the surface of silica. This material is highly active and selective for the oxidation of organic compounds to the oxygenated products (19).

Individual clusters of metal atoms can be deposited onto a substrate using the tip of an STM as a molecular paint brush (20). The atoms to be deposited are first electroplated on to the tip of the STM and then transferred to the supporting surface by contacting the tip with the surface.

Carbon nanotubes offer interesting possibilities as supports for metal particles. Such structures can be grown in the form of bundles. Recent research has demonstrated that Ir nanoparticles deposited into such tube bundles are more effective for the decomposition of hydrazine than Ir supported on a conventional support such as alumina (21). This advance is of potential interest for hydrazine-fueled thrusters used on space vehicles.

This brief overview has shown that the activity and selectivity of catalyst nanoparticles is strongly dependent on their size, shape, and surface structure, as well as their bulk and surface composition. Advances in physical methods for characterizing the structure and composition of such nanoparticles are contributing to a molecular-level 
understanding of the structure-performance relationships. Advances have also occurred in the synthesis of nanoparticles and nanoporous supports with controlled size and shape. This ongoing progress is rapidly enabling catalyst researchers in academe and industry to achieve the goal of catalysis by design.

\section{References}

1. Chem. Ind., Jan. 21, 2002, p. 22.

2. C. B. Duke, E. W. Plummer, Ed. Frontiers in Surface and Interface Science (North-Holland, Amsterdam, 2002).

3. R. Maisel. Principles of Adsorption and Reaction on Solid Surfaces (Wiley, New York, 1996).

4. G. C. Bond, D. T. Thompson. Catal. Rev. Sci. Eng. 41, (1999).

5. M. Valden, X. Lai, D. W. Goodman. Science 281, 1647 (1998).

6. T. Blasko, J. M. Lopez Nieto. Appl. Catal. A 157, 117 (1997).

7. K. Chen, A. T. Bell, E. Iglesia. J. Catal. 209, 35 (2002).

8. H. Topsoe, B. S. Clausen, F. Massoth in Catalysis: Science and Technology Vol. 10, J. R. Anderson and M. Boudart, Eds. (Springer, New York, 1996).

9. T. Koyama, Z. Weng-Sieh, R. Gronsky, F. Engleke, T. S. King, M. Pruski, A. T. Bell. J. Catal. 149, 142 (1994); J. Catal. 150, 400 (1994).

10. P. L. Gai. Topics in Catal. 21, 161 (2002).

11. M Weyland. Topics in Catal. 21, 175 (2002).

12. G. Durscher, N. D. Browning, S. J. Pennycook. Phys. Stat. Sol. (a) 166, 327 (1998).

13. F. Besenbacker, I. Chorkendorff, B. S. Clausen, B. Hammer, A. Molenbroek, J. K. Norskov, I. Stentsgaard. Science 279, 1913 (1998).

14. P. C. Thune, J. Loss, D. Wonter, P. J. Leustra, J. W. Niemantsverdriet. Macromol. Symp. 173, 37 (2001).

15. Y. Iwasawa. J. Catal, in press.

16. R. Schögl, M. Salmeron. Personal communication. 
17. C. Nozkaki, C. G. Lugmair, A. T. Bell, T. D. Tilley. J. Am. Chem. Soc. 124, 13194 (2002).

18. J. Corker, F. Lefebvre, C. Lecuyer, V. Defaud, F. Quignard, A. Cholpin, J. Evans, J.-M. Basset. Science 271, 966 (1996).

19. V. Vidal, A. Theolier, J. Thivolle-Cazat, J.-M. Basset. Science 276, 99 (1997).

20. D. Kolb. Surf. Sci. 500, 722 (2002).

21. K. P. De Jong, J. W. Geus. Catal. Rev. Sci. Eng. 42, 481 (2000). 


\section{Figure Captions}

Fig. 1 Illustration of the placement of nanoparticles in automotive catalytic converters.

Fig. 2 Effects of particle size on the activity of titania-supported gold for the oxidation of $\mathrm{CO}(5)$.

Fig. 3 Influence of titania overlayers on the turnover frequency for $\mathrm{CO}$ hydrogenation, $\mathrm{N}_{\mathrm{CO}}$, the turnover frequency for methane formation, $\mathrm{N}_{\mathrm{Cl}}$, and the probability of chain growth, $\alpha(9)$.

Fig. 4 TEM image of gold atoms on the (100) surface of nickel and quantum chemical calculations of the effects of gold atoms on the adsorption of carbon atoms (13).

Fig. 5 Molecular precursor routes to well-defined, active, single-site catalysts isolated iron atoms on silica (17). 


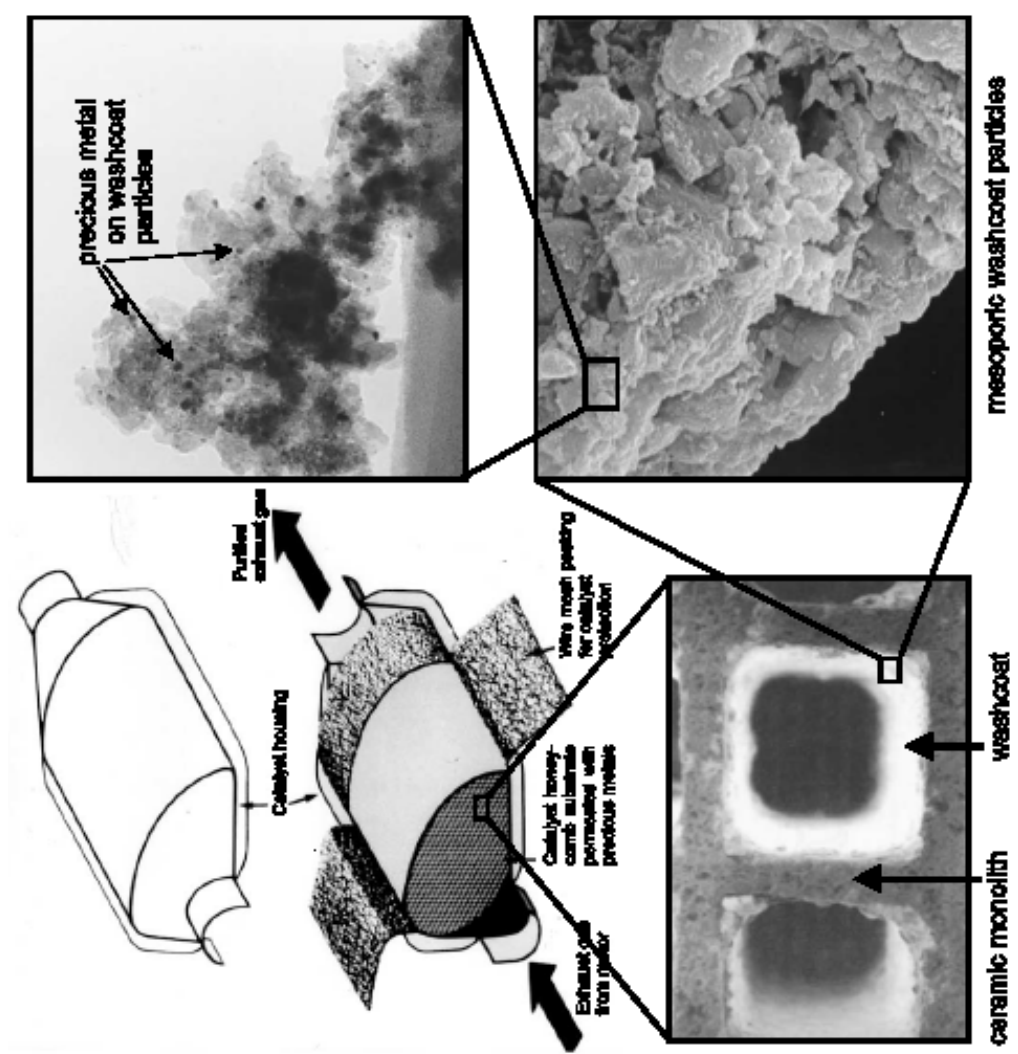



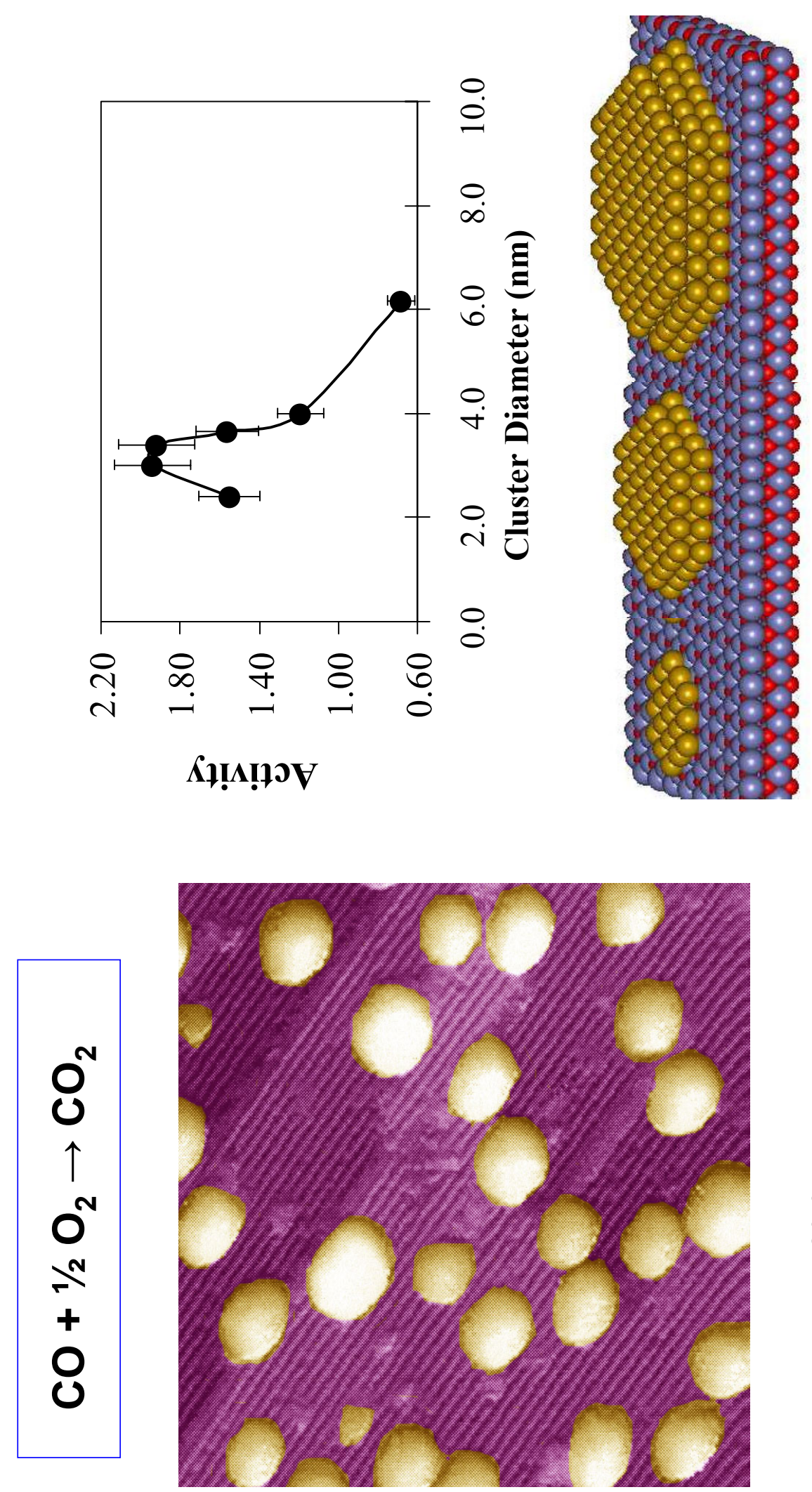

$\stackrel{\Xi}{\Xi}$

uU $0^{\circ} 0 \varepsilon$ 

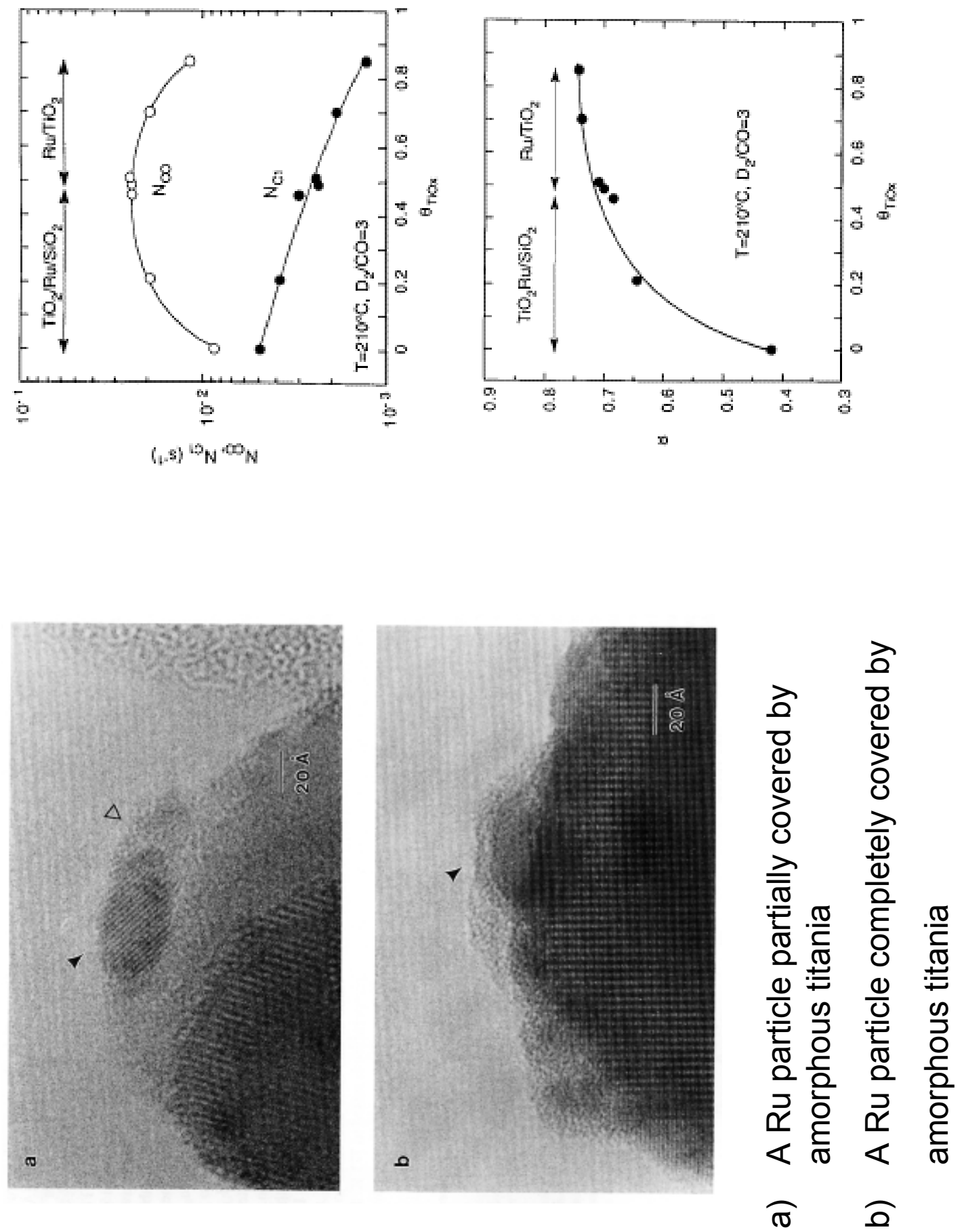


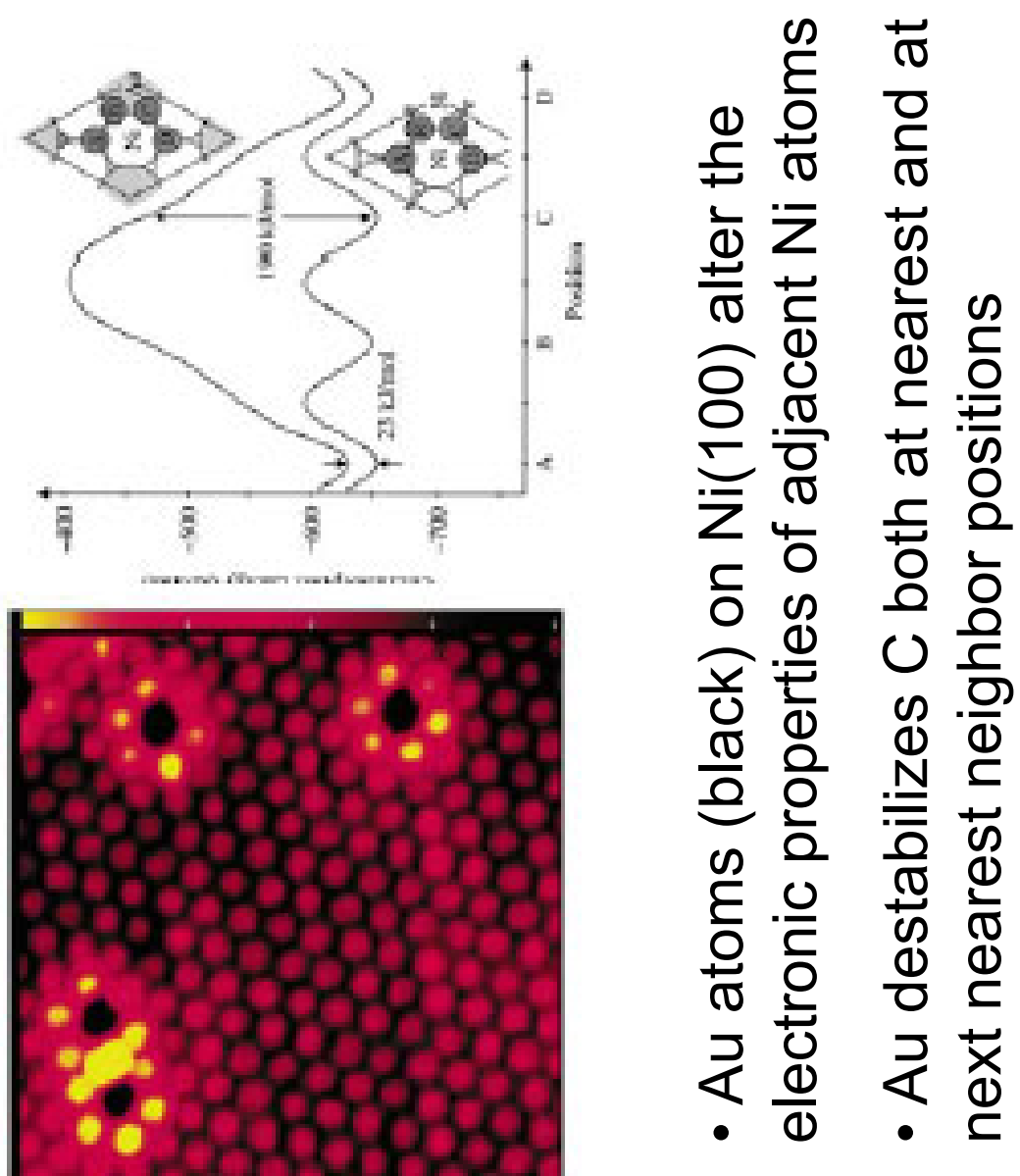




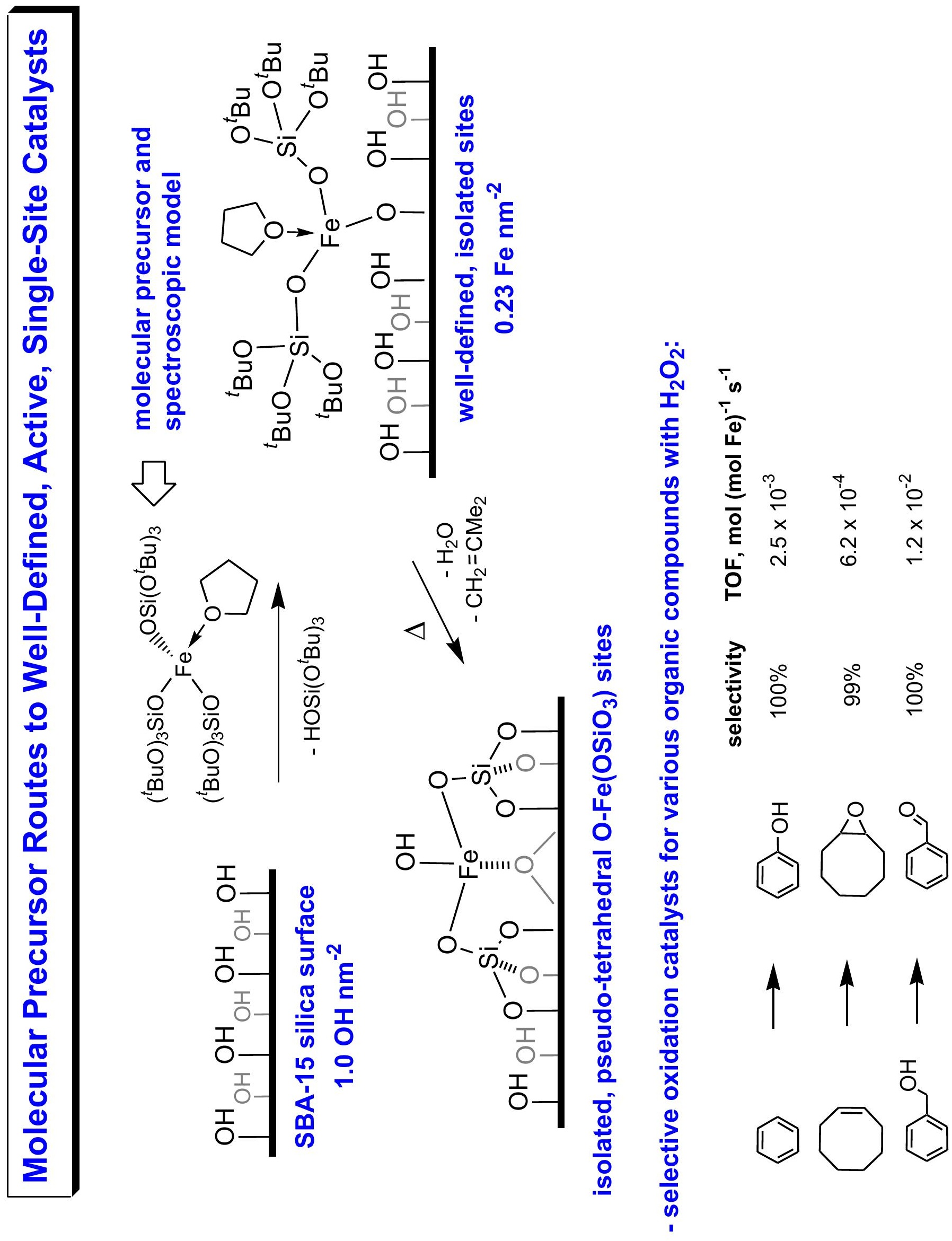

